\title{
Numerical Simulation of Internal Gravity Waves Using a Lattice Gas Model
}

\author{
J. M. Buick*, W. J. Easson** and C. A. Greated* \\ * Department of Physics and Astronomy \\ $* *$ Department of Mechanical Engineering \\ The University of Edinburgh \\ The Kings Building \\ Mayfield Road \\ Edinburgh \\ EH9 3JZ \\ UK
}

\begin{abstract}
Internal waves are modelled in two different circumstances: in a continuously stratified fluid and at the interface between two immiscible fluids. This is done using the lattice gas approach. The standard single-phase model and an immiscible two-phase model are both modified to incorporate gravitational interactions. Standing internal waves are set up in both models and are seen to oscillate under the action of the gravitational interaction. The results obtained suggest that the lattice-gas approach can be a useful tool in the modelling of such phenomena.
\end{abstract}

Key words: Lattice gas, Cellular automata, Wave Modelling, Internal Waves Standing waves.

\section{Introduction}

\subsection{Internal Waves}

Internal waves occur in two circumstances: on a continuous density gradient and on a density step at the interface of two fluids.

\section{Continuously Stratified Fluids}

Continuous density gradient internal waves can occur in any stratified fluid and are most commonly found in the ocean and in the atmosphere where a density gradient is produced. In the ocean the density is a function

$$
\rho=\rho(p, T, \chi)
$$

of the pressure $p$, the temperature $T$ and the salinity $\chi$, defined as the proportion by mass of the dissolved salts. The composition of the dissolved salts also varies but not by enough to have a significant effect on the density. In the ocean we 
expect the temperature to vary between the freezing point of sea water, $271 \mathrm{~K}$ and a maximum values of about $300 \mathrm{~K}$ giving a density change of about $0.5 \%$ The salinity varies from about $\chi=0.034$ to $\chi=0.037$ giving a density change of about $0.2 \%$ [1]. In the ocean it is found that the variation of $T$ and $\chi$ is much more important than the density changes produced by variations in the pressure $p$ with depth [1]. Thus we are in fact interested in the variable

$$
\rho_{a}(T, \chi)=\rho\left(p_{a}, T, \chi\right)
$$

the density that sea water at temperature $T$ and salinity $\chi$ would have if it was brought to atmospheric pressure $p_{a}$ without changing $T$ or $\chi$. In general we expect $\rho_{a}$ to vary between about 1020 and $1030 \mathrm{~kg} \mathrm{~m}^{-3}$, a variation of only about $1 \%$.

In the atmosphere density gradients are produced primarily by pressure variations; here the pressure variations can cause very large density differences. At the earth the pressure is atmospheric pressure $p_{a}$, this pressure decreases with increased altitude and approaches zero at the limit of the atmosphere. The variation in the density in the atmosphere can be much greater than the density variations in the ocean.

\section{Density Steps}

Interfacial internal waves can occur whenever two fluids of different densities come into contact with an interface between them. Step-like density stratifications which approximate to the two miscible fluid situation can be found in the ocean. This can occur when fresh water rivers flow into the salt water of the sea. The fresh water settles on top of the salt water and interfacial waves can be formed at the boundary. This happens in practice in many deep estuaries such as the Norwegian fjords provided conditions are such that there is no extensive mixing of the two layers. Mixing can be caused by strong tidal motion and also by rough seas.

\subsection{Lattice gas modelling}

The lattice gas model has been used successfully over the past few years to model a number of fluid phenomena ranging from simple single fluid simulations such as flow round plates [2] and channel flow [3] to more complicates flows involving two or more fluids including the Kelvin-Helmholtz instability [4], the combustion of gases [5] and wave motion at a free surface [6]. Lattice gas models have a number of advantages over more traditional numerical methods, particularly where fluid mixing and phase transitions can occur. The simulation is always performed on a regular grid and can be efficiently implemented on a massively parallel computer. Solid boundaries and multiple fluids can be introduced in a straightforward 
manner and the simulation is performed equally efficiently regardless of the complexity of the boundary or interface. The efficient implementation of complex boundaries has already been exploited in the study of flow through porous media $[7,8]$ where the porous media is represented by a random configuration of boundaries. It is the fast, parallel implementation, which the lattice gas is ideally suited to, and, when interfacial waves are being considered, the simplicity with which the interface is simulated, which make the lattice gas model a good choice for internal wave simulations.

\section{The Standard FHP Model}

The lattice gas method on a hexagonal lattice was first introduced by Frisch, Hasslacher and Pomeau (FHP) [9]. Here we consider the FHPIII model which consists of an ensemble of fluid 'particles' moving on an underlying hexagonal grid. Each particle moves along one of the six links $d_{i}(i=1,6)$ where the direction of $d_{i}$ is given by $\sin \left(\frac{\pi i}{3}-\frac{\pi}{6}\right) \boldsymbol{i}+\cos \left(\frac{\pi i}{3}-\frac{\pi}{6}\right) \boldsymbol{j}$, where $\boldsymbol{i}$ and $\boldsymbol{j}$ are unit vectors along the orthogonal $x$ - and $y$-axis shown in figure 1 , or remains at rest at one of the intersection points (sites) of the lattice, link $d_{0}$. The link directions and the coordinate system are shown in Figure 1. The following constraints are applied to the motion of the particles:

1. Only one particle is allowed on each link at one time; this is referred to as the exclusion principle.

2. Particles on link $d_{0}$ have zero velocity and all other particles travel at unit speed moving from one site to a neighbouring site in each time-step. A particle travelling on link $d_{i}$ has velocity $\boldsymbol{e}_{i}$, where $\left|\boldsymbol{e}_{i}\right|=1$.

3. At each time-step the particles at each site collide in such a way that the number of particles and the momentum are conserved at each site.

These constraints make the FHP model very suitable for computer implementation. The model is naturally discretized in space and time since only the state of each site at each time-step is required to fully describe the system and to calculate the state of the system at the next time-step. The restriction of only one particle on each link means that the state of each site can be represented by one 7-bit number $s=\left(n_{0}, n_{1}, n_{2}, n_{3}, n_{4}, n_{5}, n_{6}\right)$ where $n_{i}$ is one if there is a particle on link $d_{i}$ and zero otherwise. Updating the model between time-steps is also efficient since the new state of each site at any time-step depends only on its own state and the state of its six nearest neighbours at the previous time-step and the outcome of the particle collisions. Since there are only 7 possible links for the particles to travel on and a maximum of 7 particles allowed at each site, there 
are only ever a maximum of two possible outcomes which conserve both particle number and momentum. There is no need to calculate these outcomes at each site at each time-step; they are found using a look-up table. Any set of collision rules can be used provided they conserve particle number and momentum. Here we use the FHPIII collisions which are formed from the basic collisions shown in Figure 2 and their rotations through $\pm 60^{\circ}$, the basic collisions with a spectator particle (moving or at rest) and the dual of these collisions (found by swapping full and empty links). In Figure 2 the left-hand column represents the particles approaching the site before collision. The right hand column represents the outcome of the collision. Where there are two possible outcomes one is picked at random. Rest particles are represented by a solid sphere. Provided the FHP collisions satisfy the conservation of mass and momentum equations

$$
\sum_{i} n_{i}\left(t+1, \boldsymbol{r}+\boldsymbol{e}_{i}\right)=\sum_{i} n_{i}(t, \boldsymbol{r})
$$

and

$$
\sum_{i} \boldsymbol{e}_{i} n_{i}\left(t+1, \boldsymbol{r}+\boldsymbol{e}_{i}\right)=\sum_{i} \boldsymbol{e}_{i} n_{i}(t, \boldsymbol{r})
$$

at each site, it can be shown $[10,9]$ to satisfy the equations

$$
\partial_{t} \rho+\sum_{i} \partial_{i}\left(\rho u_{i}\right)=0
$$

and

$$
\partial_{t}\left(\rho u_{i}\right)+\sum_{j} \partial_{x_{j}}\left[\rho g(d) u_{i} u_{j}\right]=-\partial_{x_{i}} P+\sum_{j} \partial_{x_{j}}\left[\nu(\rho) \partial_{j} u_{i}\right]
$$

where $\rho$ is the density, $P$ is the pressure, $d$ is the density per link, $d=\rho / 7$ and $\nu$ is the viscosity. For the FHPIII model [10]

$$
g(d)=\frac{7(1-2 d)}{12(1-d)}
$$

and

$$
\nu=\frac{1}{28} \frac{1}{d(1-d)} \frac{1}{1-8 d(1-d) / 7}-\frac{1}{8} .
$$

These are the continuity equation and the Navier-Stokes equation with an extra factor $g$ which is a function of the density and of the collision rules used. The viscosity, $\nu$, is also a function of the density and the collision rules. The NavierStokes equation can be recovered by rescaling the velocity and pressure by $g$ [11]: $u^{\prime} \rightarrow u g, P^{\prime} \rightarrow P / g$. We then recover the Navier-Stokes equation in the scaled variables.

\section{Calculating Macroscopic Quantities}


The microscopic density $\rho$ and velocity $\boldsymbol{u}$ at each site are defined by

$$
\rho(t, \boldsymbol{r}) \stackrel{\text { def }}{=} \sum_{i} n_{i}(t, \boldsymbol{r})
$$

and

$$
\rho \boldsymbol{u}(t, \boldsymbol{r}) \stackrel{\text { def }}{=} \sum_{i} \boldsymbol{e}_{i} n_{i}(t, \boldsymbol{r})
$$

Macroscopic quantities such as the fluid velocity and density are found by dividing the grid into cells containing several lattice sites and averaging the microscopic velocity or density over the cell. The larger the cell the less noisy the results will be, however, the size of a cell is restricted by the limits imposed on the overall grid size by computer memory and time restrictions. Typically a cell will be no smaller than 16 by 16 sites. Figure 3 shows four averaging cells, each 6 sites by

Table 1: The total number of particles $\sum n_{i}$ on each link, the $x$ and $y$ components of the averaged velocity $\boldsymbol{u}$, its magnitude and inclination from the horizontal, $\theta$, the total number of particles $N$ and the average density $\rho$ for the four averaging cells shown in Figure 3.

\begin{tabular}{|l|l|l|l|l|l|l|l|l|l|l|}
\hline \hline Cell & $\sum n_{1}$ & $\sum n_{2}$ & $\sum n_{3}$ & $\sum n_{4}$ & $\mathrm{~N}$ & $\begin{array}{l}\rho \\
(\mathrm{lu})\end{array}$ & $\begin{array}{l}u_{x} \\
(\mathrm{lu})\end{array}$ & $\begin{array}{l}u_{y} \\
(\mathrm{lu})\end{array}$ & $\begin{array}{l}|\boldsymbol{u}| \\
(\mathrm{lu})\end{array}$ & $\begin{array}{l}\theta \\
(\mathrm{deg})\end{array}$ \\
\hline $\mathrm{A}$ & 6 & 3 & 4 & 3 & 16 & $16 / 36$ & 0 & $2 / \rho$ & $2 / \rho$ & 90 \\
$\mathrm{~B}$ & 5 & 3 & 5 & 3 & 16 & $16 / 36$ & 0 & 0 & 0 & $\sim$ \\
$\mathrm{C}$ & 4 & 4 & 3 & 3 & 14 & $14 / 36$ & $1 / \rho$ & $1 / \rho$ & $\sqrt{2} / \rho$ & 45 \\
$\mathrm{D}$ & 4 & 5 & 3 & 3 & 15 & $15 / 36$ & $2 / \rho$ & $1 / \rho$ & $\sqrt{5} / \rho$ & 26.5 \\
\hline \hline
\end{tabular}

6 , on a portion of a square lattice (a square lattice has been used for convenience to demonstrate the averaging principle). Table 1 shows the microscopic details of the total number of particles $\sum n_{i} i=1, . ., 4$ on each link and the total number of particles in each cell, $N$. Also shown in the table are the details of the macroscopic velocity $\boldsymbol{u}$ and the macroscopic density $\rho$ which are derived from the microscopic quantities. The angle $\theta$ is the angle between the velocity direction and the $x$-axis. Note that the density is defined to be the mean number of particles per site in the cell rather than the mean number of particles per unit volume and the velocity is defined as the vector sum of all the microscopic velocities.

\subsection{Colour Models}

Two different fluids can be modelled by labelling the particles according to the fluid they belong to. Thus a 'red' and a 'blue' fluid can be simulated at the 
same time provided the number of red particles, $\sum_{i} n_{i}^{(r)}$, and the number of blue particles, $\sum_{i} n_{i}^{(b)}$, are conserved at each time-step. This is achieved if the conservation of colour equations

$$
\begin{aligned}
& \sum_{i} n_{i}^{(r)}\left(t+1, \boldsymbol{r}+\boldsymbol{e}_{i}\right)=\sum_{i} n_{i}^{(r)}(t, \boldsymbol{r}) \\
& \sum_{i} n_{i}^{(b)}\left(t+1, \boldsymbol{r}+\boldsymbol{e}_{i}\right)=\sum_{i} n_{i}^{(b)}(t, \boldsymbol{r})
\end{aligned}
$$

are added to the conservation of mass and momentum equations (1) and (2). Provided these quantities are conserved there is no need to further restrict the particles after a collision. Thus if a red and a blue particle collide head-on then they will collide according to the collision rule shown in the first row of Figure 2, one of the two outcomes being picked at random as in the single-particle model. To allow for the conservation of colour all that is required is that after the collision one of the particles is red and one is blue. This can be achieved either by randomly selecting which is red and which is blue or using some other scheme. If the random method is used then the two fluids will mix together, however different methods can be used to assign the colour and these will influence the behaviour of the model.

\section{The Colour-Field Model}

The colour-field surface tension model was devised by Rothman et al. [12]. It specifies a method for distributing the colour after a collision (subject to the conservation laws) which causes the two fluids to fully separate and produces a surface tension between them. Let $C_{i}(t, \boldsymbol{r})$, the colour density of link $d_{i}$ at time $t$ and site $\boldsymbol{r}$, be given by

$$
C_{i}(t, \boldsymbol{r}) \stackrel{\text { def }}{=} n_{i}^{(r)}(t, \boldsymbol{r})-n_{i}^{(b)}(t, \boldsymbol{r})
$$

the difference between the number of red and blue particles on the link. Due to the exclusion principle $C_{i}$ can take the values $-1,0$ and 1 . The colour density at a site is given by

$$
C(t, \boldsymbol{r})=\sum_{i} C_{i}(t, \boldsymbol{r})
$$

The local colour flux $\boldsymbol{q}\left[s^{(r)}(t, \boldsymbol{r}), s^{(b)}(t, \boldsymbol{r})\right]$ is given by

$$
\boldsymbol{q}\left[s^{(r)}(t, \boldsymbol{r}), s^{(b)}(t, \boldsymbol{r})\right] \stackrel{\text { def }}{=} \sum_{i} C_{i}(t, \boldsymbol{r}) \boldsymbol{e}_{i}
$$

and is the difference between the red momentum and the blue momentum at $\boldsymbol{r}$ at time $t$ where the site is in state $s=s^{(r)}+s^{(b)}$, the sum of the red and blue states. The local colour field $\boldsymbol{f}(t, \boldsymbol{r})$ is given by

$$
\boldsymbol{f}(t, \boldsymbol{r}) \stackrel{\text { def }}{=} \sum_{i} \boldsymbol{e}_{i} \sum_{j} C_{j}\left(t, \boldsymbol{r}+\boldsymbol{e}_{i}\right)
$$


Table 2: The possible outcomes of a head-on collision between one red and one blue particle

\begin{tabular}{|l|l|l|}
\hline \hline & $s^{\prime \prime(r)}$ & $s^{\prime \prime(b)}$ \\
\hline (a) & $(0,1,0,0,0,0,0)$ & $(0,0,0,0,1,0,0)$ \\
(b) & $(0,0,0,0,1,0,0)$ & $(0,1,0,0,0,0,0)$ \\
(c) & $(0,0,0,1,0,0,0)$ & $(0,0,0,0,0,0,1)$ \\
(d) & $(0,0,0,0,0,0,1)$ & $(0,0,0,1,0,0,0)$ \\
(e) & $(0,0,1,0,0,0,0)$ & $(0,0,0,0,0,1,0)$ \\
(f) & $(0,0,0,0,0,1,0)$ & $(0,0,1,0,0,0,0)$ \\
\hline \hline
\end{tabular}

which is the microscopic gradient of the colour density $C(t, \boldsymbol{r})$. The work $W\left(s^{(r)}, s^{(b)}\right)$ performed by the flux against the field is

$$
W\left(s^{(r)}, s^{(b)}\right)=-\boldsymbol{f} \cdot \boldsymbol{q}\left(s^{(r)}, s^{(b)}\right) .
$$

The out-state of any FHP interaction $s^{(r)} \rightarrow s^{(r)}, s^{(b)} \rightarrow s^{\prime(b)}$ is then chosen such that

$$
W\left(s^{\prime(r)}, s^{\prime(b)}\right)=\min _{s^{\prime \prime(r)}, s^{\prime \prime(b)}} W\left(s^{\prime \prime(r)}, s^{\prime \prime(b)}\right)
$$

where $s^{\prime \prime(r)}, s^{\prime \prime(b)}$ are all the possible collision outcomes. Here the effect of the colour field model is maximised by not only considering $s^{\prime \prime(r)}, s^{\prime \prime(b)}$ to be the possible outcomes of any collision but also by considering the possibility of no FHP collision taking place and the (colourless) particles continuing in a straight line. Consider the first collision shown in Figure 2 where one particle is red and one is blue, we can represent $s^{(r)}$ and $s^{(b)}$ by $s^{(r)}=(0,0,1,0,0,0,0)$ and $s^{(b)}=(0,0,0,0,0,1,0)$ (a red particle in link 2 and a blue particle in link 5$)$. The possible outcomes are shown in Table 2 where (a) and (b) are the two colour distributions possible for the first outcome shown in Figure 2, (c) and (d) are the two distributions possible for the second outcome shown in Figure 2 and (e) and (f) are possible distributions if no collision takes place. The outcome selected $\left(s^{\prime(r)}, s^{\prime(b)}\right)$ is the one which minimises $W$. The effect of this additional interaction is to separate the two fluids with a definite interface between them. Other than the labelling of the particles, the two fluids are treated in the same way and so both fluids have the same properties.

\subsection{Gravitational Interactions in the FHP Model}

To simulate a gravitational force we need to introduce an interaction which will decrease the fluid momentum in the vertical direction while preserving the momentum in the horizontal direction. This was done in the basic FHP model 
by flipping a small number of particles from link $d_{1}$ to $d_{3}$ and from link $d_{6}$ to $d_{4}$ provided there were no particles already on the destination links. With this implementation of gravity the $x$-direction is horizontal and the $y$-direction is vertical. The strength of such an interaction depends on the number of flips which take place each time-step. We ensure that the number of such particle flips is small compared to the number of FHP collisions so that the additional interaction, while producing a noticeable effect, will only have a negligible affect on the other properties of the fluid [13]. There are two parameters which describe the gravitational interaction. The first is $S_{g}$ which is the percentage of all possible gravity flips which are performed. The other is $\mu$ which is the mean number of flips per site per time-step, this is measured during the simulation.

In a two-particle model gravitational interactions were introduced in a similar manner, however when two fluids of different 'densities' were being considered it was required that the interaction strength be greater for the 'heavier' fluid then for the 'lighter' fluid. This was done either by having the gravitational interaction acting only on the heavier fluid or by having it acting on both fluids but with different strengths.

\subsection{Boundary Conditions}

Two different boundary conditions can be applied at the edge of the grid. A solid boundary can be applied which is either no-slip or free-slip. A no-slip boundary reflects the particles back along the direction they approach in. A free-slip boundary reflects particles so that their momentum parallel to the boundary is conserved and their momentum perpendicular to the boundary is reversed. In the simulations described here all the free-slip boundaries are along the directions of the $x$-axis. Thus a particle approaching the solid boundary along link $d_{1}$ is reflected back along link $d_{3}$. These solid boundary conditions can also be applied in the interior of the grid to simulate solid objects. The other kind of boundary condition applied here is the continuous boundary condition. This is always applied at the edge of the grid and acts so that particles moving off the grid at one edge move onto the grid at the opposite edge. Thus a particle on a $N_{x}$ by $N_{y}$ grid with position $\left(N_{x}, y\right)$ and velocity $\boldsymbol{e}_{2}$ at time $t$ will move to position $(1, y)$ at time $t_{1}$ if a continuous boundary is applied at $x=1$ and $x=N_{x}$.

\subsection{Errors in a Lattice Gas Model}

The lattice gas model is different in several ways from the more traditional numerical methods which have been applied to fluid simulations. There is also a marked difference in the way errors appear in the results. The model involves 
tracking particles as they move and collide according to simple rules. This is implemented using integer or Boolean arithmetic so there are no errors introduced by, for example, rounding errors or finite difference approximations to derivatives; sources of error which appear in other numerical techniques. The absence of such errors means that changing the grid size does not affect the accuracy of the results in the way it would if a traditional numerical method was being applied. In fact, changing the grid size changes the problem which is being simulated. Consider, for example, the simulation on a $N_{x}$ by $N_{y}$ grid of a wave with wavelength $\lambda=N_{x}$. If the simulation is repeated on a $2 N_{x}$ by $2 N_{y}$ grid then either the wavelength is kept the same and two wavelengths of the wave are simulated or the wavelength of the new wave becomes $2 N_{x}$ and one wavelength is simulated as before. In the first case there is no change in the resolution of either of the two wavelengths being simulated (although an ensemble average could be performed over the two wavelengths). The second case describes the action which would normally be taken in a numerical simulation to reduce the error, here it changes the wave which is being simulated in the same way that doubling the wavelength of a wave produced in a wave tank would change the wave period and velocities. This has been shown to be the case for surface waves [6] and also applies to the internal waves being considered here.

The errors in measurements made from a lattice gas simulation come from the size of averaging cell used, not the grid size. The error in the density and velocity measurements are given by [14]

$$
\Delta \rho=1.128 \sqrt{\frac{d(1-d)}{7 S}}
$$

and

$$
\Delta u=\frac{C(\rho)}{\sqrt{S}}
$$

where $S$ is the number of sites which are averaged over and $C(\rho)$ is no larger than 0.43 . It should be noted that the source of this error is the measuring technique and so the error is the same at all times: it does not accumulate over time. To reduce the error the averaging process must be over as many sites as possible. The size of the averaging cell is restricted since is must be small compared to the typical length scale over which the simulation is changing. Temporal averaging is also possible, again, provided the averaging takes place over a time significantly smaller than the smallest time scale of the simulation. Ensemble averaging can also be performed between different simulations of the same problem. 


\section{Numerical Simulations}

A number of simulations were performed on the CM-200 at Edinburgh University for both internal waves in a stratified fluid and interfacial waves at a density step. The stratified fluid was produced using the FHPIII model with the additional gravitational interaction; a horizontal density step was produced using the colour-field model with the additional gravitational interaction. The gravitational interaction is required here because we are simulating gravity waves. In each case a horizontal boundary was placed at the bottom and top of the computational grid and a continuous boundary was used at the other edges. The grid size used varied for the different simulations but in every case the wave amplitude was considerably smaller than its wavelength. Standing waves were simulated throughout because of the simplicity with which they can be set up. An initial experiment was run in which the fluid is initially given zero average velocity and allowed to settle under the action of the gravitational interaction. This gave the density profile which we would expect when no waves were present. A small sinusoidal standing wave at the extreme of its oscillation (the wave velocities all zero) was then superimposed to give the initial conditions for the wave simulations. These waves were then allowed to oscillate under gravity. At prescribed times during the simulation the velocity and density of the fluid(s) were found by averaging the microscopic quantities.

\section{Results and Discussion}

\subsection{Internal Waves in a Stratified Fluid}

A 2048 by 1024 site grid was initialised with an average density of $\rho_{0}=2.1$ particles per site and zero velocity with a horizontal free-slip boundary at the top and bottom edges and with continuous boundary conditions on the other edges. The system was then allowed to evolve under the FHPIII collision rules and the gravitational interaction with strength $S_{g}=0.15$ for 3,000 . The gradient across the stratified fluid was found by averaging the density across each of the rows. This is shown in Figure 4 where the density gradient is seen to be constant across the fluid. A sinusoidal wave was then superimposed on the density gradient as described in section 2 . The system was allowed to evolve and the velocity and density were measured by averaging over 32 by 16 site cells every 1,000 time-steps. The height of the central wave was found from each density result by counting the number of cells with density greater than $\rho_{0}$ in each of the 64 columns. This method only gives the height to the nearest averaging cell, however given the noise in the density, this is an acceptable method. These heights were then Fourier transformed to find the mean height and amplitude of the wave. Figure 5 shows the height of the central wave at its centre, calculated from its 
mean height and amplitude, plotted against time. Also shown in Figure 5 is the best-fit damped cosine curve of the form $A e^{-\alpha t} \cos (2 \pi t / \tau+\phi)+h$ with the fitted parameters shown in Table 3 . The best-fit curve shows a good agreement with the data. $A$ is the initial amplitude of the wave, $\alpha$ is the damping constant, $\tau$ is

Table 3: The best fit data for the curve in Figure 5. All units are in terms of lattice units and time-steps.

\begin{tabular}{|c|c|c|c|c|c|}
\hline \hline $\mathrm{A}$ & $\alpha$ & $\tau$ & $h$ & $\phi$ & $\epsilon$ \\
\hline 19.52 & $-1.14 \times 10^{-5}$ & 3122 & 439 & -0.0246 & 1.271 \\
\hline \hline
\end{tabular}

the wave period, $h$ is the mean depth and $\phi$ is a phase shift which is introduced to account for any initial time during which the set-up wave settles down to its natural form before it starts to oscillate. The variable $\phi$ allows for any error in the set up of the wave, we expect it to be small if the wave has been initialised properly. All units measurements are in lattice units (lu), that is the units of length and time are the lattice spacing and the time-step. The root mean square deviation between the data points and the curve is given by $\epsilon$. The attenuation is seen to be negligible over the first 10,000 time-steps ( 3 periods) and the best-fit decay constant $\alpha$ was found to be small and negative. There will be some error in the fitted parameters here due to the small number of data points and also due to them being only accurate to the scale of the averaging cells. Clearly $\alpha$ must be positive but very small.

The density profile across the whole wave at times $t=3,000$ and $t=8,000$ are shown in Figures 6 and 7 . These figures show the density distribution of the fluid when the wave is approximately at each extreme of its motion. The density measurements are slightly noisy due to the random factors involved in the model and the size of the averaging cell. Despite the noise, a sinusoidal density variation can clearly be seen across the fluid at each height, the amplitude of the variation being seen to be approximately constant at all heights.

Figure 8 shows the $x$-velocity distribution of the fluid at $\mathrm{t}=4,000$ time-steps averaged over 64 by 32 site cells and extrapolated using a bilinear extrapolation routine. This has the effect of further reducing the noise. The interpolated figure shows two main features:

1. the $x$-velocity distribution in the $x$-direction has a sinusoidal variation as expected; 
2 . There is a small variation of the $x$-velocity in the $y$-direction, the magnitude of the velocity being slightly larger at the bottom of the wave than at the top.

Similar results are found for the $x$-velocity at the other times when results were taken, with the magnitude of the velocity dependent on the phase of the wave. The error in the velocities, given by equation (5), is \pm 0.009 which is greater than $10 \%$ of the maximum extrapolated velocities in Figure 8. For waves with phases such that the velocities are lower the noise can become excessive. Similar results were obtained for the $y$-velocities but they were smaller and consequently noisier.

\subsection{Interfacial Internal Wave Simulation on a Density Step}

Internal waves on a density step were implemented using the colour-field model and the gravitational interaction for waves of wavelength $2048 \mathrm{lu}$. The simulation was run for two cases: case (a) where gravity acted only on the heavy particles with a strength $S_{g}=0.15$ and case (b) where the gravitational interaction acts on the heavy particles with strength $S_{g}=0.2$ and the light particles with strength $S_{g}=0.1$. In both cases the grid was initialised by first setting up a system with zero average velocity and with an average density of 4.9 particles per site. This is the density at which the colour-field rules give optimal separation of the two fluids [12]. The grid was then divided into two sections with a horizontal line separating them; the bottom section slightly larger than the top section. Particles in the bottom section were then coloured red and the other particles coloured blue. The system was then allowed to evolved for 2000 time-steps under the required gravitational interaction and the colour-field surface tension rules and the height of the interface between the two fluids found.

The density of the two fluids is shown in Figure 9 for case (a) where the density has been found by averaging over each row. Figure 9 shows a small but constant density gradient across the heavy fluid as is expected, since gravity acts in this fluid. The lighter fluid also has a constant, but smaller, density gradient across it despite the fact that gravity is not acting on it. This was seen to be a general feature and independent of the value of $S_{g}$ in the heavy fluid. This is produced in the interface region where the gravitational interaction reduces the vertical momentum of the heavy particles. This momentum change is passed on, to some extent, to the lighter particles during the implementation of the colour-field rules. Increasing $S_{g}$ in the heavy fluid increased the density gradient in the heavy fluid but left the density gradient in the lighter fluid unchanged. Figure 9 also shows what appears to be a large mixed area between the two fluids where there are significant numbers of both particles. In reality this is simply due to the inter-

face not being completely horizontal; the interface between the two fluids (where 
both particles are present) is rarely more than one of two sites thick. For case (b) a similar graph was obtained but here the density gradients of both fluids depended on their value of $S_{g}$. A new sinusoidal interface was drawn in place of the horizontal interface and all the particles re-coloured red if they were below the interface and blue if they were above it. For both simulations the height of the wave at its centre was found every 40 time-steps. This was done by considering a central column 16 sites wide. The number of rows containing mainly red particles was found in the column and also the number of rows containing mainly blue particles. The results are shown for cases (a) and (b) in Figures 10 and 11 respectively, along with the best-fit curves as before. A good likeness is seen between the data and the best-fit curves. The values found for the best-fit parameters are given in Table 4.

The results for case (a) show the wave amplitude $A$, the damping constant $\alpha$ and

Table 4: The best fit parameters for the curves in Figures 10 and 11. All units are in terms of lattice units and time-steps.

\begin{tabular}{|l|l||c|c|c|c|c|c|}
\hline \hline case & fluid & $\mathrm{A}$ & $\alpha$ & $\tau$ & $h$ & $\phi$ & $\epsilon$ \\
\hline (a) & red & 22.92 & $4.290 \times 10^{-5}$ & 14,633 & 220 & -0.2348 & 3.74 \\
$(\mathrm{a})$ & blue & -24.04 & $4.431 \times 10^{-5}$ & 14,512 & 233 & -0.2741 & 4.07 \\
(b) & red & 27.22 & $8.680 \times 10^{-5}$ & 21,870 & 222 & -0.0753 & 3.38 \\
(b) & blue & -33.12 & $1.048 \times 10^{-4}$ & 18,810 & 230 & -0.136 & 2.95 \\
\hline \hline
\end{tabular}

the period $\tau$ for the two fluids agreeing to within a few percent and the phase factor $\phi$ is only a few percent of a period, suggesting that the wave was initialised well. The mean variation, $\epsilon$, between the curve and the data is also small, 3 or 4 lattice units, suggesting a good fit. The values of $\epsilon$ obtained for case (b) are no larger then the ones obtained for case (a), suggesting the curve is as good a fit, although the best-fit parameters obtained for the red and blue fluids differ slightly more for case (b) than case (a). Comparing the values of the periods and the damping constants obtained for the two cases considered, it is seen that the period and the damping constant are larger for case (b). The larger period is case (b) suggests that the effective gravitational strength depends on the average number of particles flipped in both fluid and not on the absolute number in either. The mean number of particles flipped per site per time-step, $\mu$, for the two simulations are shown in Table 5. The change in the damping constant is due to the different densities of the fluids in the two simulations. The results also show that the sum of the mean depths, $h$, for both simulations is slightly greater than the grid size. This can be explained by the surface between the two fluids not 
Table 5: The mean number of gravity flips per site per time-step for the two simulations

\begin{tabular}{|c||c|c|}
\hline \hline case & $\begin{array}{c}\text { mean flips in } \\
\text { heavy fluid }\end{array}$ & $\begin{array}{c}\text { mean flips in } \\
\text { light fluid }\end{array}$ \\
\hline (a) & $1.33 \times 10^{-3}$ & - \\
(b) & $1.40 \times 10^{-3}$ & $8.35 \times 10^{-4}$ \\
\hline \hline
\end{tabular}

being completely flat and also the fluids settling down slightly at the start. This is also reflected in the difference between the values of $A$ found for both fluids.

Velocity plots at times $t=4,000$ and $t=10,000$ are shown in Figures 12 and 13 for case (a). These vector plots show the motion of the two fluids. The thick arrows represent cells in which at least $95 \%$ of the particles are heavy particles while the thinner arrows are for cells containing only light particles and also the cells at the boundary which are partially filled with both particles. The difference in the depth of the two fluids seen in Figures 10 and 11 can also be seen here. The wave motion can be observed in both of the fluids as can an area between the two fluids where the velocities are small. This area is particularly noticeable when the fluid velocities are largest. It is not clear from the results whether this is due to the motion of the two fluids travelling in opposite directions at the boundary causing a small boundary layer to be set up or whether it is due to the averaging cell containing particles travelling in both directions. The velocities obtained are small, many being less than 0.01 and so they are fairly noisy. The wave motion is however distinct and its clarity can be improved either by using an ensemble averaging technique [6] or a filtering technique. The velocities of the red and blue fluids were also recorded for case (b) and show the same features, although the velocities tend to be smaller due to the increased damping.

\section{Discussion}

Two different approaches have been used to simulate internal waves. Internal waves on a continuous density gradient were modelled using the FHPIII model with additional gravitational interactions. Internal waves on a density step were modelled using an FHPIII model with two distinct particle types by introducing colour-field surface tension interactions and gravitational interactions. 


\subsection{Internal Waves in a Stratified Fluid}

The ability of a lattice gas model to perform such simulations was demonstrated. The density and velocity profile of the wave were found at selected times. The wave motion could be seen clearly in the density plots and the velocity plots also showed evidence of wave motion although they were some what noisier. The wave was seen to damp only slowly; over three periods there was no noticeable decay.

\subsection{Interfacial Internal Waves on a Density Step}

Internal waves on a density step were also simulated. It has been seen that waves can be produced provided one fluid is made 'heavier' than the other. It was shown that this difference in the strength of the gravitational interaction can be simulated by applying the gravitational interaction with different strengths to the two fluids, where the lower strength can be zero. The velocities of these internal waves were found to be relatively small and therefore fairly noisy, however they are clearly seen to describe wave motion in both the fluids. The noise in the results can be reduced using ensemble averaging techniques.

\subsection{Comparison of models}

One major difference between the results obtained for the two different types of internal waves studied was the size of the damping constant. The density of the two fluids at the interface is about 4.9 particles per site which, because of the duality of the model (swapping particles and empty links in one collision rule produces another allowed collision), means the viscosity of the fluid should be the same as the viscosity of a fluid with density $7-4.9=2.1$ particles per site. This is the density which was used to model waves in the stratified fluid. The colour-field collision rules affect on the fluid when there are particles of different colours present at the same site. This only occurs in a small number of sites on either side of the fluid interface so the additional interaction should have only a minimal affect on the viscosity of either fluid. A small increase in the viscosity of the colour-field fluid is expected however [12], because the in-state is allowed to be the same as the out-state even when a collision can occur. A higher damping rate is observed in practice for interfacial waves when compared to other motion in a fluid with same viscosity. One feature of the FHP model is that the viscosity can be large even at a density of 2.1 particles per site, the density where it is minimum for the FHPIII collision rules, so we would expect some damping due to viscous effects. These effects have been minimised by using the FHPIII collision rules which give the lowest viscosity of any of the standard rules and by using large wavelengths which have the effect of increasing the Reynolds number of the 
simulation.

\subsection{Comparison of Results with Experiment and Theory}

Standing internal waves have been studied experimentally by Thorpe [15]. He performs experiments on standing interfacial waves and standing waves in a stratified fluid using a tank 14 inches long fitted with two plungers, one at either end, a fixed distance above the tank bottom. His photographic results [15] show the same features as the results obtained here. For the stratified fluid his results show a sinusoidal variation in the density at all depths. The size of the variation is reduced slightly at the top and bottom of the tank due to the boundaries. Other than this the density variations shown in Figures 6 and 7 compare well with the experimental results. The results obtained here for the interfacial internal waves also compare well to Thorpe's experimental results for low amplitude waves.

A qualitative comparison can be made between the results obtained and their expected theoretical values which are well established $[1,16]$. This is done for the wave on a continuously stratified fluid and for the interfacial wave when gravity acts on both fluids. For both waves we expect the maximum horizontal velocity to be $u^{\prime}=\omega a$ where $a$ is the wave amplitude at the time the velocities were measured and $\omega$ is the wave frequency $\omega=2 \pi / \tau$. Here $u^{\prime}$ is the theoretical velocity and is related to the measured velocity through the scaling relation: $u=\omega a / g$. The frequency of the wave in the continuously stratified fluid is the Väisälä-Brunt frequency:

$$
\omega=\sqrt{\frac{-G}{\rho} \frac{\mathrm{d} \rho}{\mathrm{d} z}},
$$

where $G$ is the acceleration due to gravity. The rate of damping is

$$
\alpha=2 k^{2} \nu
$$

The rate of damping and the frequency of an interfacial wave, in deep water, is given by $[17]$

$$
\omega=\omega_{0}-\omega^{\prime}
$$

and

$$
\alpha=\omega^{\prime}+\alpha^{\prime}
$$

where

$$
\begin{aligned}
& \omega_{0}=\sqrt{G k \frac{(f-1)}{(f+1)}}, \\
& \omega^{\prime}=\omega_{0}^{1 / 2} \frac{\sqrt{2} k f \sqrt{\nu}}{(1+f)^{2}}
\end{aligned}
$$


and

$$
\alpha^{\prime}=2 k^{2} \frac{\nu\left(1+f^{3}\right)}{(1+f)^{3}}
$$

where $\rho$ is the density of the lighter fluid and $f \rho$ is the density of the heavier fluid. To find $G$ we consider the total force acting on $S$ sites containing $M$ particles (each of unit mass) at a given time. The total force is $M G=S \rho G$. This is equal to the change in momentum which is $S \bar{\mu} \sqrt{3} g$ since each particle flip changes a particles measured speed by $\sqrt{3}$ and its scaled speed by $\sqrt{3} g$. The value of $\bar{\mu}$ is taken to the average of the two values of $\mu$ in the two fluids. Equating these two expressions gives

$$
G=\frac{\sqrt{3} \bar{\mu} g}{\rho}
$$

where $\mu$ is taken to be the average of the value in each fluid for the interfacial wave. The value of $\rho$ is taken to be its mean value and $\nu(d)$ and $g(d)$ are calculated from equations (4) and (3) using this mean value. The ratio $f$ is the value of $\mu$ in the denser fluid divided by $\mu$ in the lighter fluid. Using these values the following theoretical values are found at $t=\tau / 4$ for the continuously stratified fluid: $\tau=3,607, \alpha=1.9 \times 10^{-6}$ and $u=0.11$; and for the two-fluid simulation: $\tau=19,300, \alpha=6.22 \times 10^{-6}$ and $u=0.017$, all in lattice units. With the exception of $\alpha$ for the interfacial wave these theoretical values are consistent with the values obtained in the simulations. The theoretical velocity is slightly larger than the maximum values shown in figure 8 , however, the velocities in figure 8 were measured at $t=4,000$ time-steps, slightly after they have their maximum value. The velocities shown in figure 12 are slightly larger than the theoretical velocity, however, figure 12 is for case (a) which is not as heavily damped as case (b). The theoretical value of $\alpha$ for the interfacial wave predicts a significantly smaller damping rate than was observed. This may partly be due to Harrison's theoretical expression [17] being a series solution in terms of $\sqrt{\nu}$ only up to order $O(\nu)$. This would not totally explain the difference between the simulation result and the theoretical value and it suggests that the wave is being damped by the action of the colour-field rules acting at the surface.

\subsection{Relating the Simulation Results to Real Physical Prob- lems}

All the results presented here are in terms of lattice units which describe the lattice gas model. It is important to be able to relate the results of a simulation to a real problem in the physical world. To do this we must compare the dimensionless parameters between the two situations. To do this we examine the dimensionless parameters describing the simulated waves. The ratio of the initial wave amplitude to the wavelength is small for each wave, it is never larger than 
0.016. This means that we are considering linear waves. The depth of the stratified fluid is half of the wavelength. The fluid depths in the two-fluid simulation is such that $k h$ has a maximum value of 0.71 . For this value $\tanh (k h)=0.61$, however, since a no-slip boundary is applied at the top and bottom of the fluid the wave can be considered as being in deep water. The Reynolds number and Froude number, defined $R e=c / k \nu$ and $c \sqrt{k / G}$ respectively where $c$ is the wave celerity $c=\omega / k$, of the two waves considered above are 2140 and 0.8 for the continuous wave and 300 and 0.5 for the interfacial wave. The ratio of the fluid densities in the two-fluid simulation is $f=1.68$. The simulations relate directly to a wave in the real world with the same or similar dimensionless numbers.

\section{Conclusion}

We have seen that the lattice gas model can be applied to simulate internal waves, both on a continuous density profile and at a density step between two immiscible fluids. The results obtained, although noisy, show the same qualitative features as waves produced in a wave tank by Thorpe [15]. The results also showed reasonable agreement with theory except for the damping rate of the interfacial wave which was significantly larger than predicted. This may be due to the action of the colour-field rules at the interface.

\section{Acknowledgements}

The authors would like to thank the Edinburgh Parallel Computing Centre (EPCC) for supplying time on the CM-200. The first author was supported by the Science and Engineering Research Council.

\section{References}

[1] J. Lighthill. Waves In Fluids. Cambridge University Press, 1978.

[2] D. d' Humières and P. Lallemand. Numerical simulations of hydrodynamics with lattice gas automata in two dimensions. Complex Syst., 1:599, 1987.

[3] D. d' Humières and P. Lallemand. Flow of a lattice gas between two parallel plates and development of the Poiseuille profile. C. R. Acad. Sci. Paris, 302:983, 1986.

[4] D. d' Humières, P. Lallemand, and G. Searby. Numerical experiments on lattice gases: Mixtures and Galilean invariance. Complex Syst., 1:633-647, 1987. 
[5] G. Searby, V Zenlé, and B Denet. Lattice gas mixtures and reactive flows. In Discrete Kinetic Theory, Lattice Gas Dynamics and the Foundations of Hydrodynamics, Torino, Italy, September 1988.

[6] J. Buick, W. Easson, and C. Greated. Simulation of wave motion using a lattice gas model. Int. J. Numer. Meth. Fluids, 22:313-321, 1996.

[7] D. H. Rothman. Cellular-automaton fluids: A model for flow in porous media. Geophys., 53:509-518, 1988.

[8] M. Sahimi. Flow phenomena in rocks: From continuum models to fractals, percolation, cellular automata and simulated annealing. Rev. Mod. Phys., 65:1393-1534, 1993.

[9] U. Frisch, B. Hasslacher, and Y. Pomeau. Lattice-gas automata for the Navier-Stokes equation. Phys. Rev. Lett., 56:1505-1508, 1986.

[10] U. Frisch, D. d' Humières, B. Hasslacher, P. Lallemand, and Y. Pomeau. Lattice gas hydrodynamics in two and three dimensions. Complex Syst., 1:649-707, 1987.

[11] S. Wolfram. Cellular automaton fluids 1: Basic theory. J. Stat. Phys., 45:471-529, 1986.

[12] D. H. Rothman and J. M. Keller. Immiscible cellular-automaton fluids. J. Stat. Phys., 52:1119-1127, 1988.

[13] J. P. Boon and A. Noullez. Lattice gas hydrodynamics. In Special Course on Modern Theoretical and Experimental Approaches to Turbulent Flow Structure and its Modelling. AGARD (Advisory Group for Aerospace Research and Development) NATO, 1987.

[14] W. Roberts. Techniques for Modelling Incompressible Fluid Flow. PhD thesis, The University of Edinburgh, 1991.

[15] S. A. Thorpe. On standing internal gravity waves of finite amplitude. $J$. Fluid Mech., 32:489-528, 1968.

[16] O. M. Phillips. The Dynamics of the Upper Ocean. Cambridge University Press, 1969.

[17] W. J. Harrison. The influence of viscosity on the oscillations of superposed fluids. Proc. Lon. Math. Soc. Series 2, 6:396-405, 1908. 

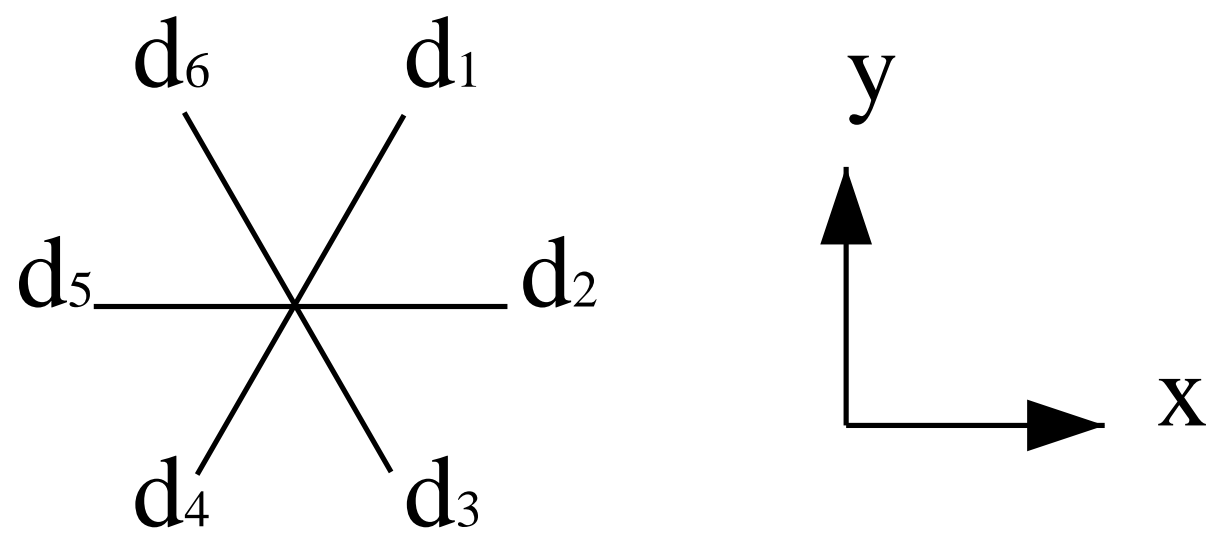

Figure 1: The six directions of the hexagonal lattice. 

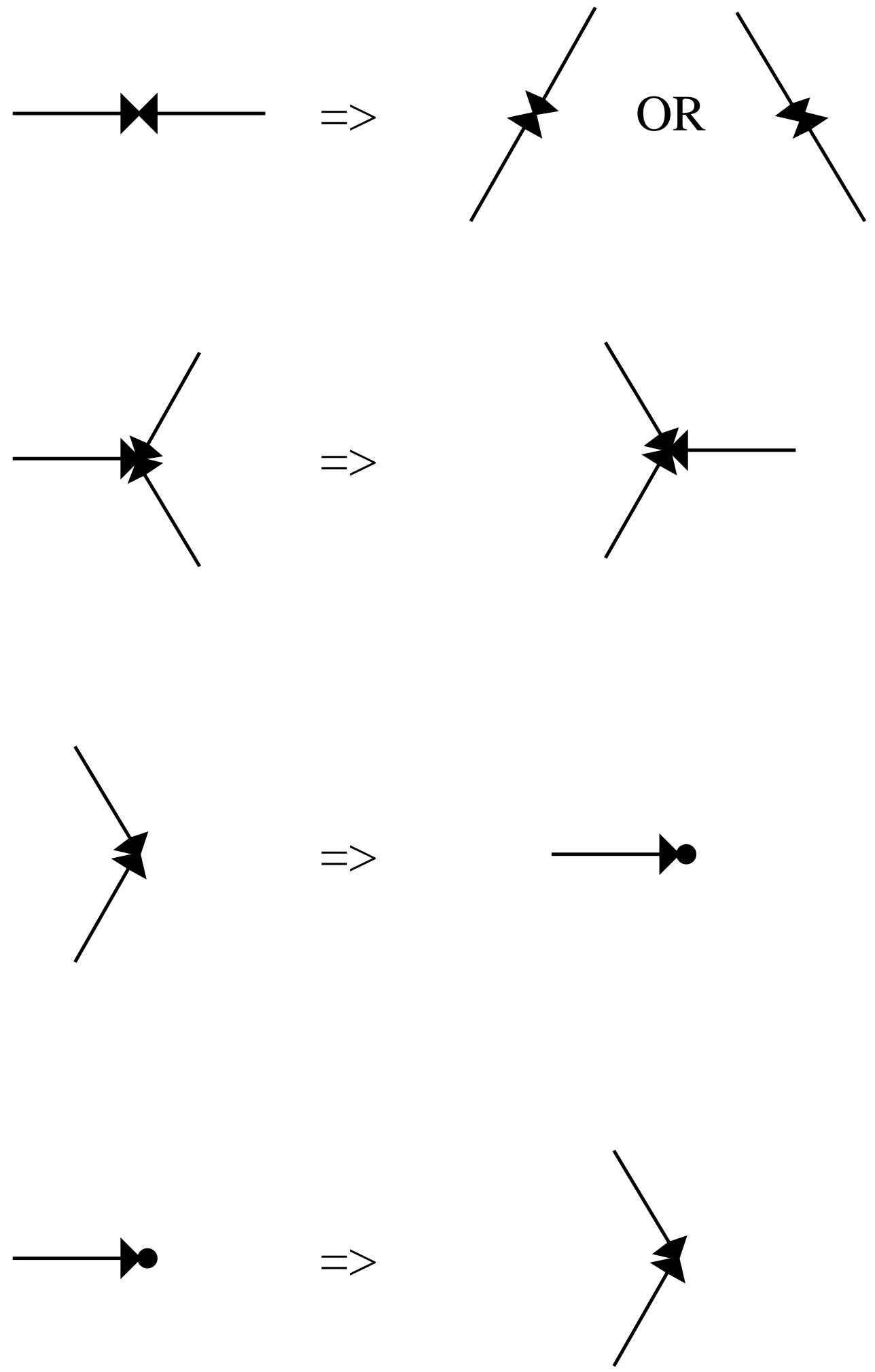

Figure 2: The basic FHP collision rules. 

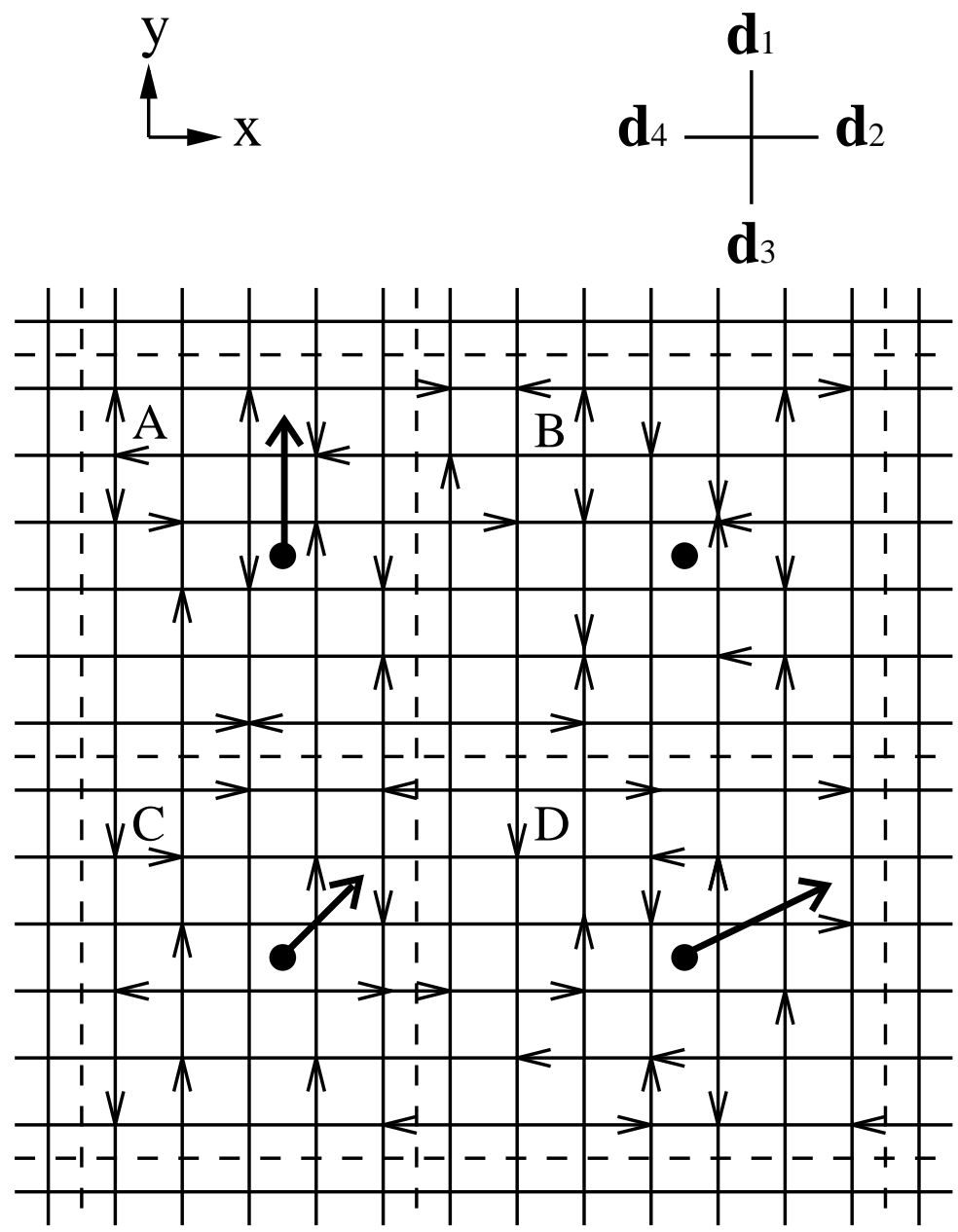

Figure 3: An example of averaging on a section of a square lattice with four averaging cells, shown by the dashed lines, superimposed over the grid. The individual particles are represented by the small arrows and the average velocities by the large arrows. The details of the different velocities and densities are shown in Table 1. 


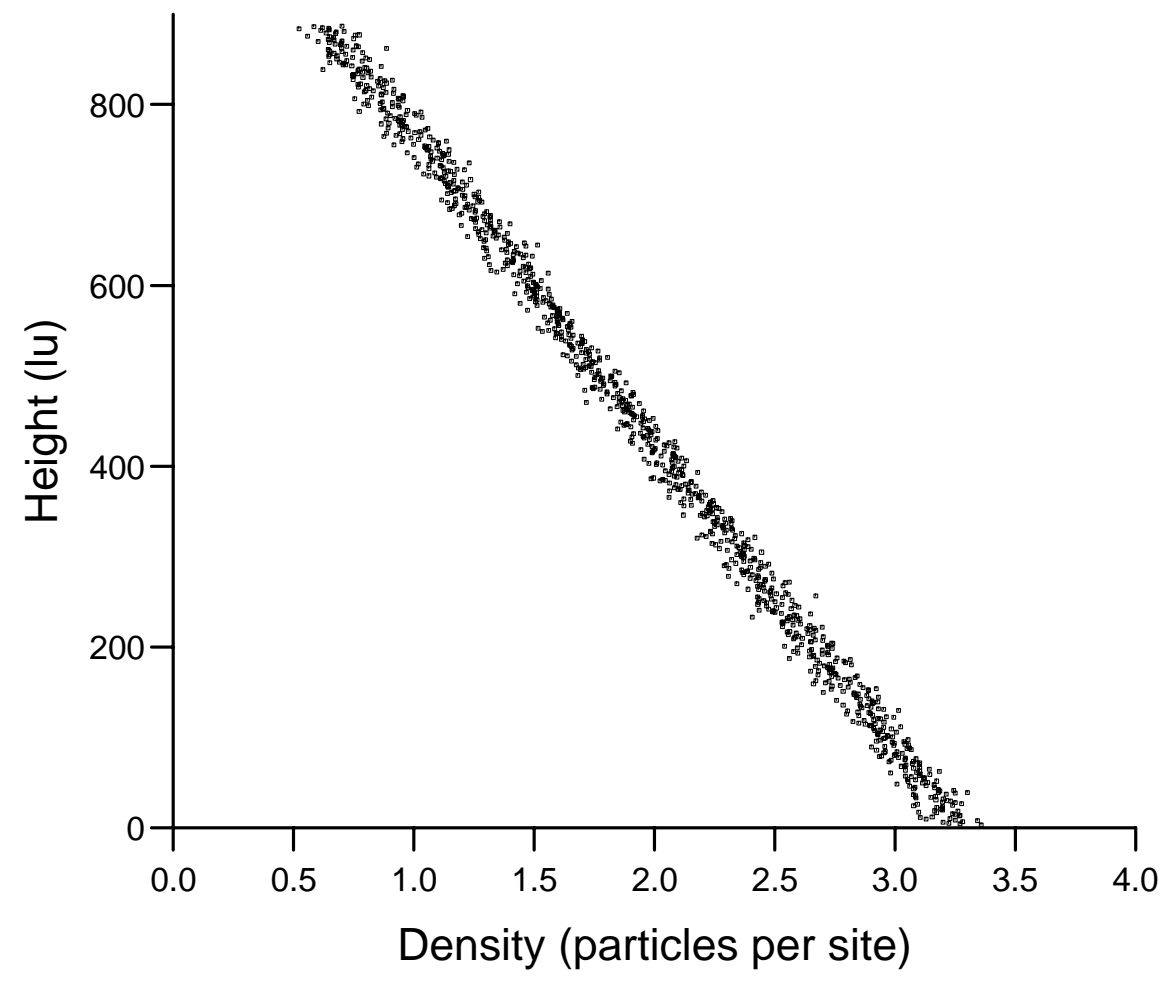

Figure 4: The variation in density with height when $S_{g}=0.15$. 


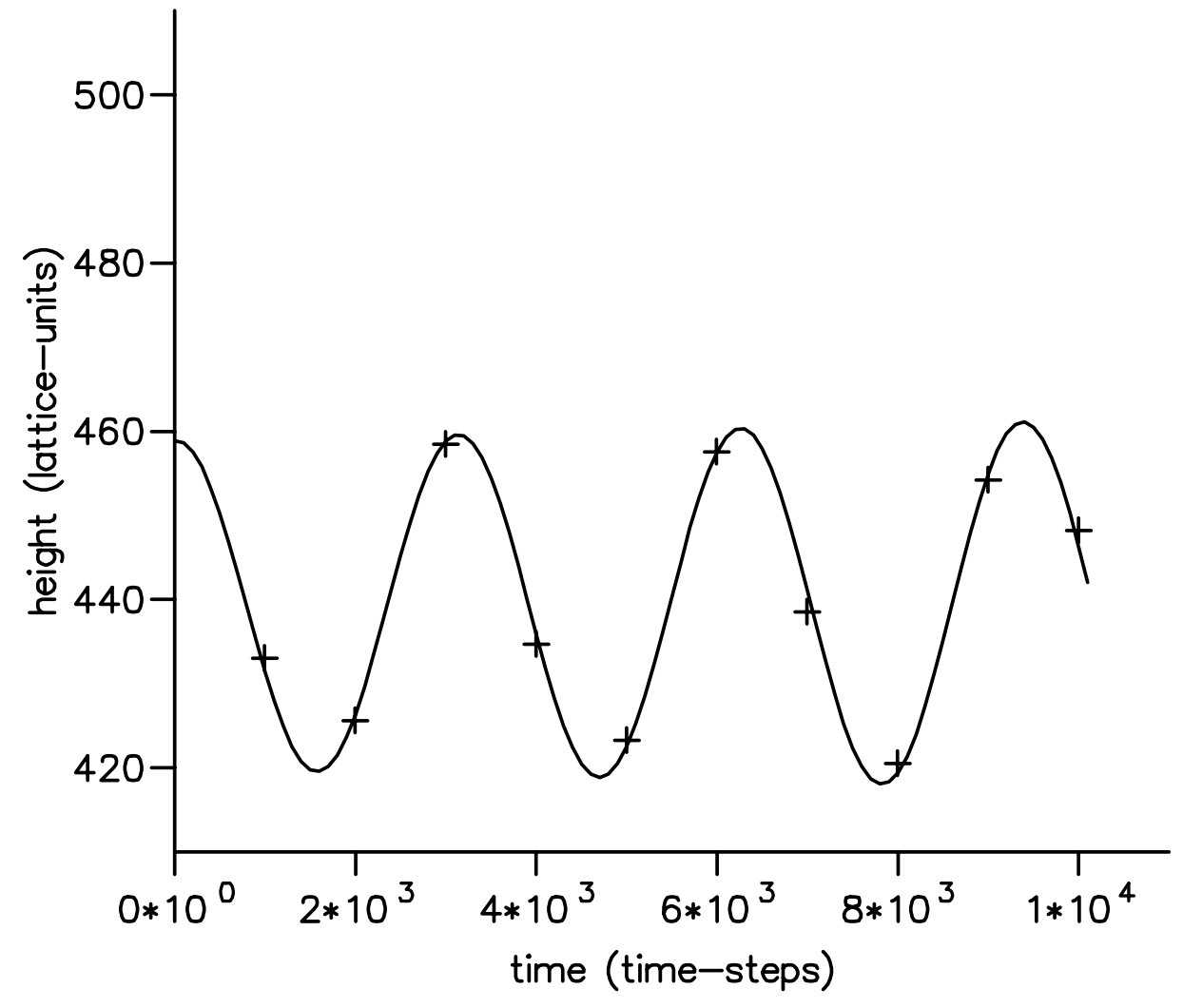

Figure 5: The height of the $\rho=2.1$ wave at its centre, plotted against time and the best-fit curve through the points. 


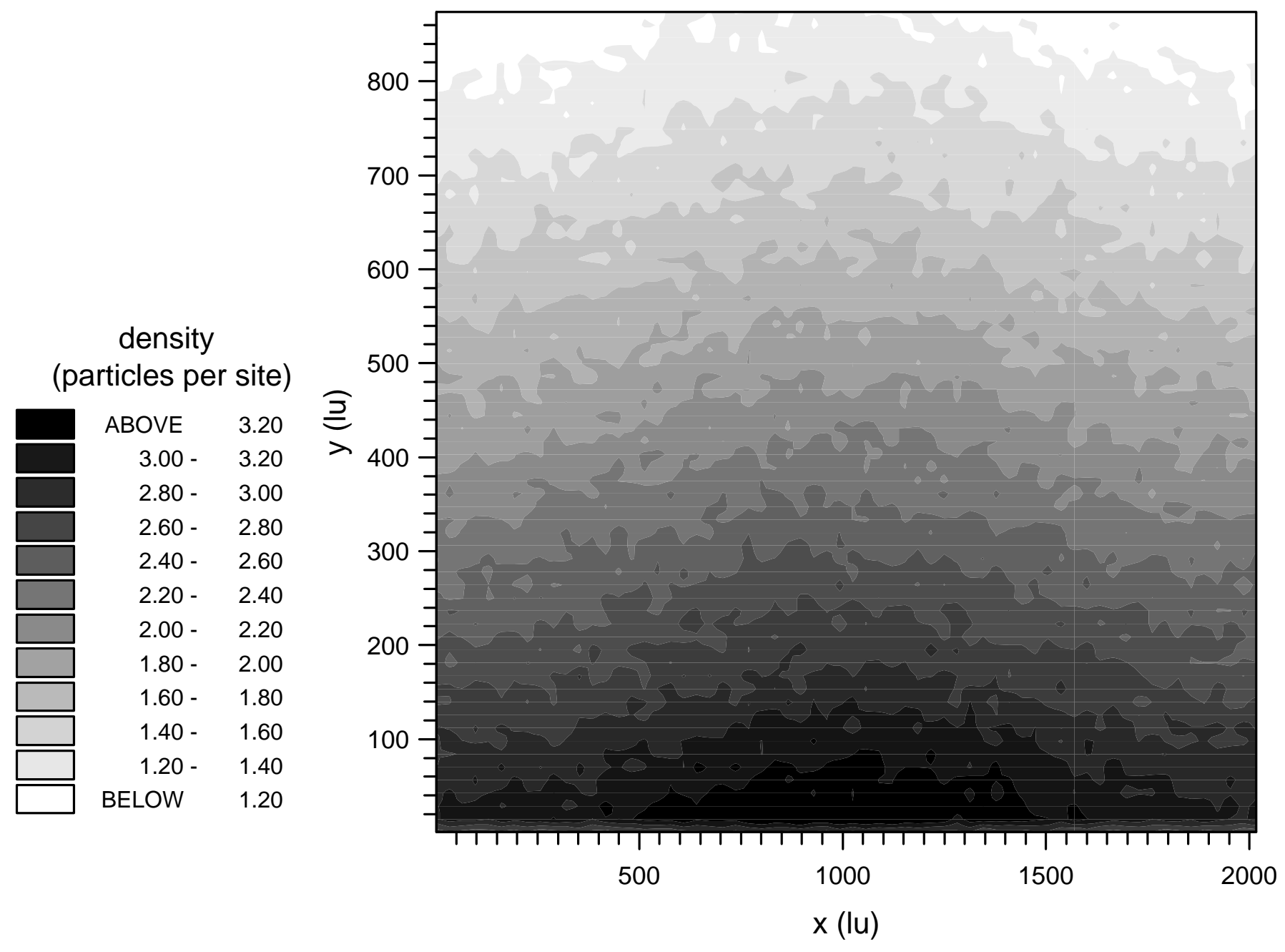

Figure 6: The density profile of an internal wave in a stratified fluid at $t=3,000$ computed using the FHPIII model. 


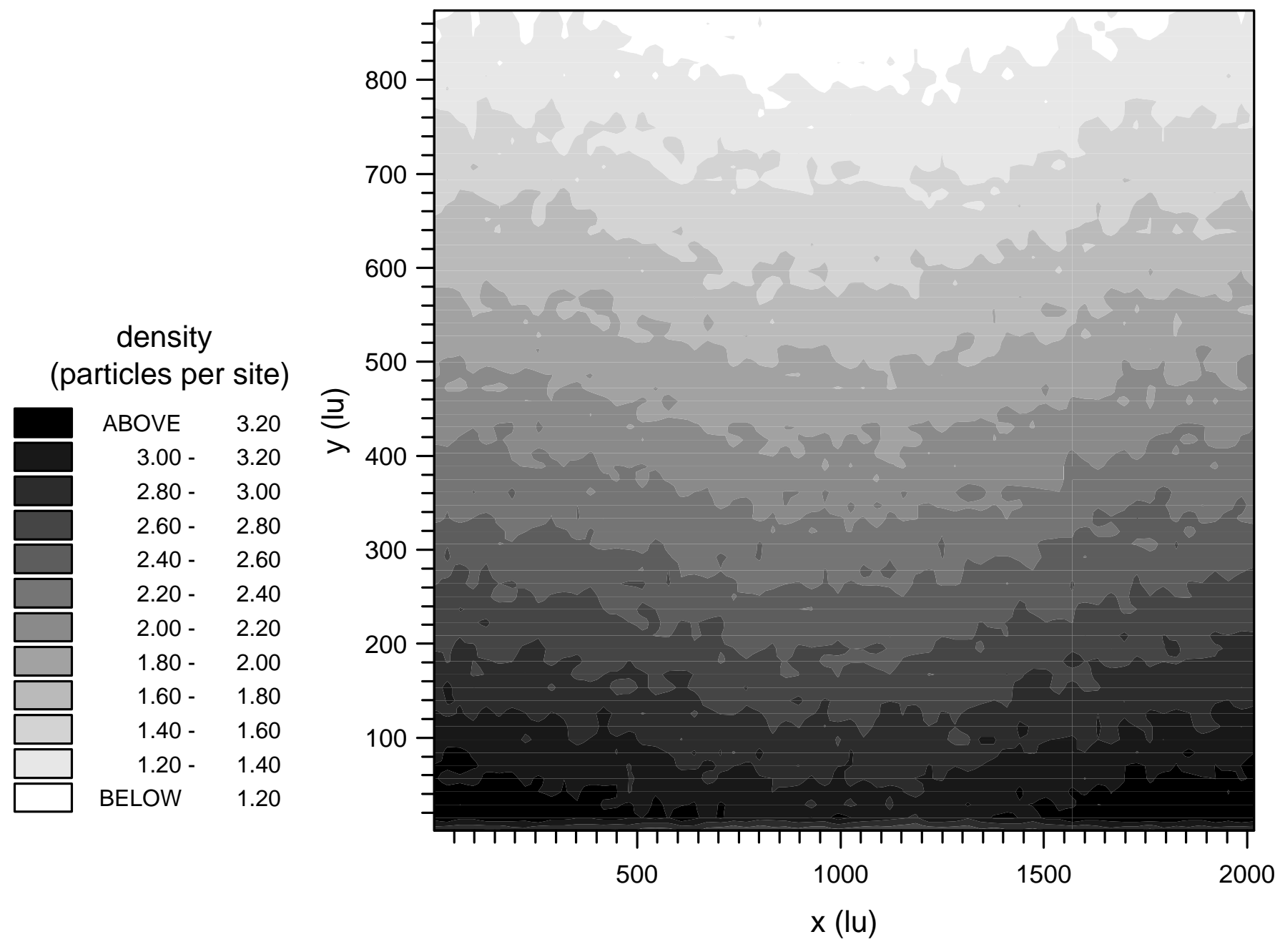

Figure 7: The density profile of an internal wave in a stratified fluid at $t=8,000$ computed using the FHPIII model. 


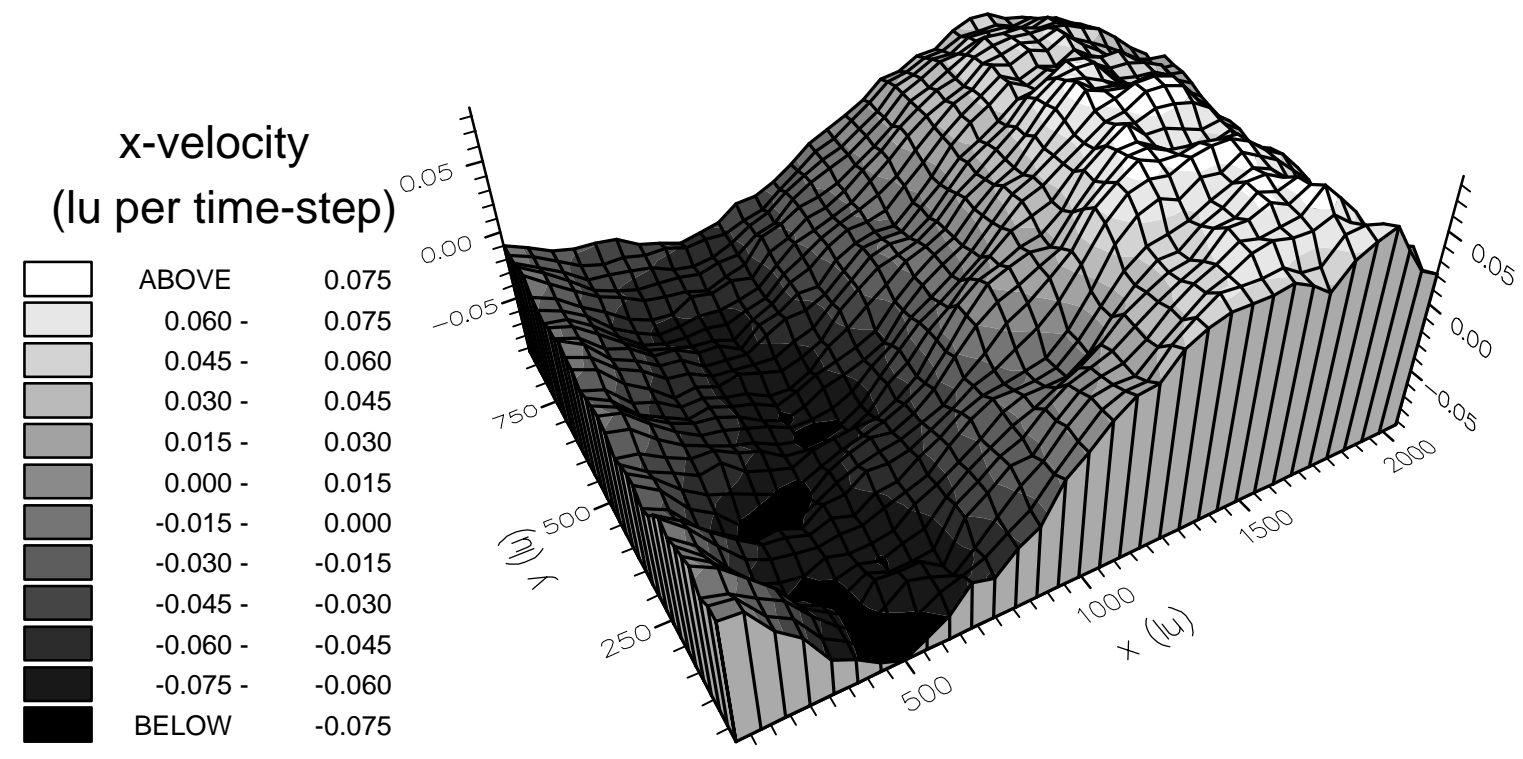

Figure 8: The extrapolated $x$-velocity distribution of an internal wave in a stratified fluid after 4,000 time-steps. 


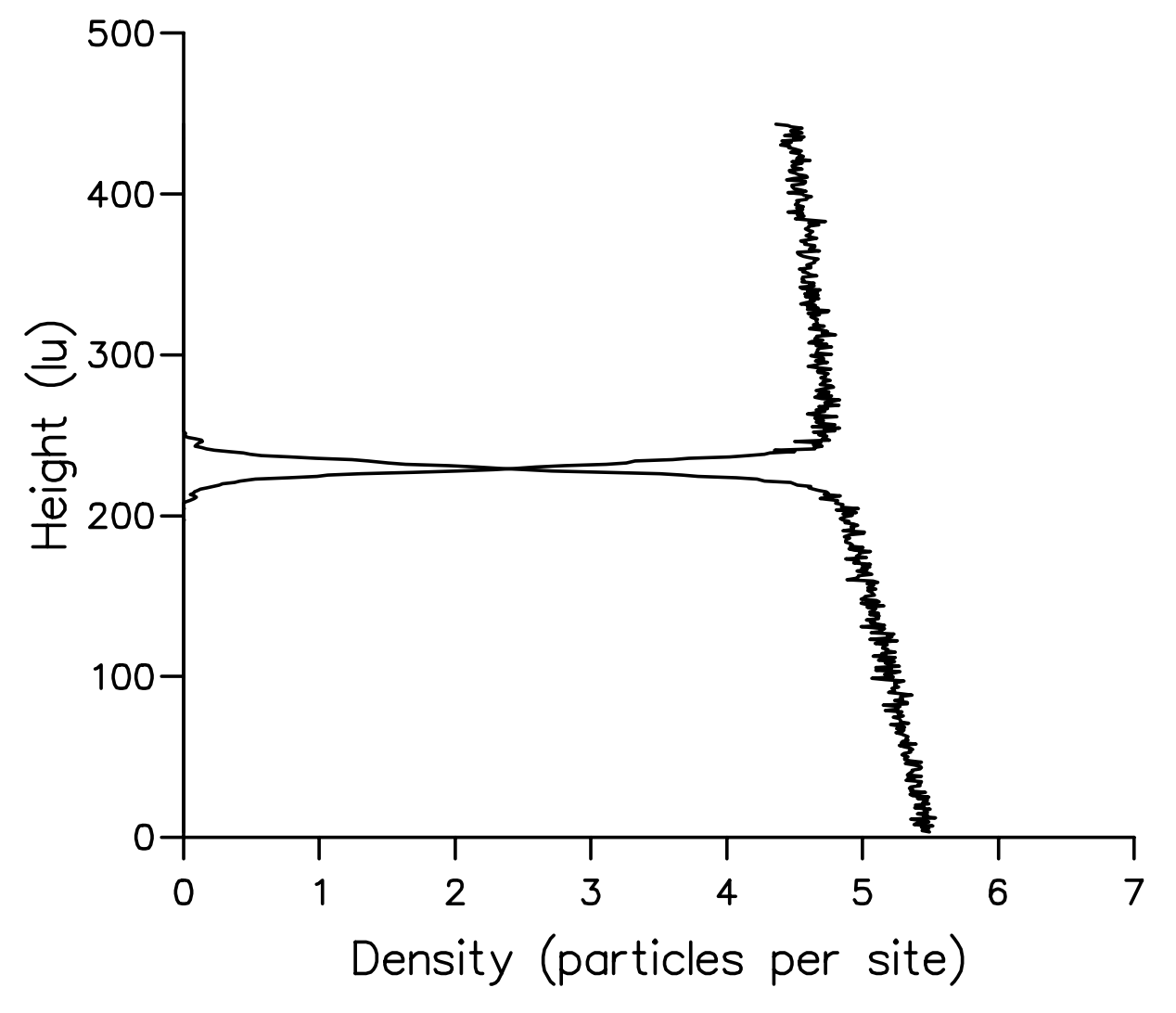

Figure 9: The variation in density with height for both fluids when gravity acts only on the heavy fluid with strength $S_{g}=0.15$ 


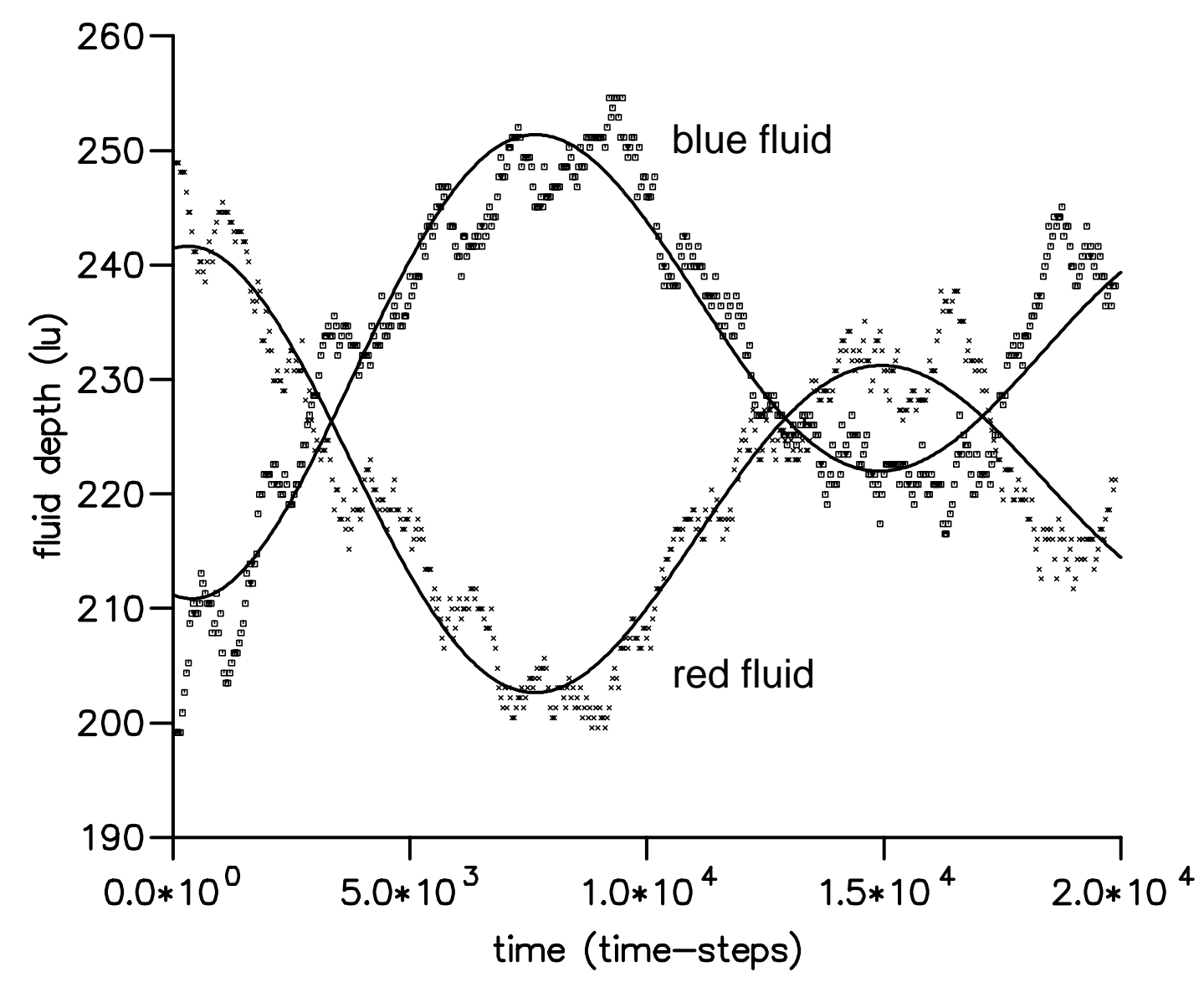

Figure 10: The fluid depth at the centre plotted against time for both the red and blue fluids and the best fit curves through both sets of data found when gravity is applied to the heavy particles only with a strength $S_{g}=0.15$. 


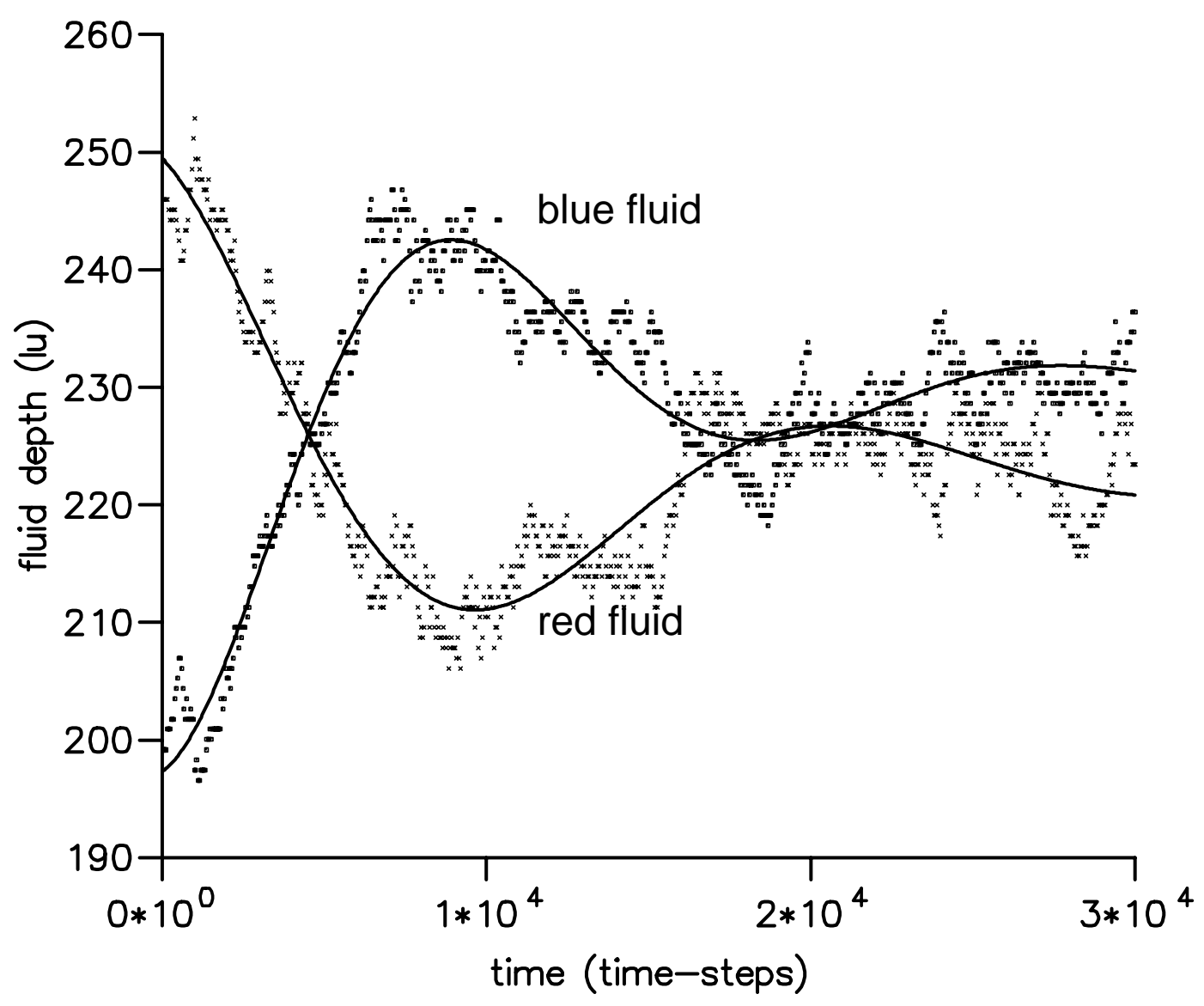

Figure 11: The fluid depth at the centre plotted against time for both the red and blue fluids and the best fit curves through both sets of data when gravity is applied to the heavy fluid with strength $S_{g}=0.2$ and to the light fluid with strength $S_{g}=0.1$. 


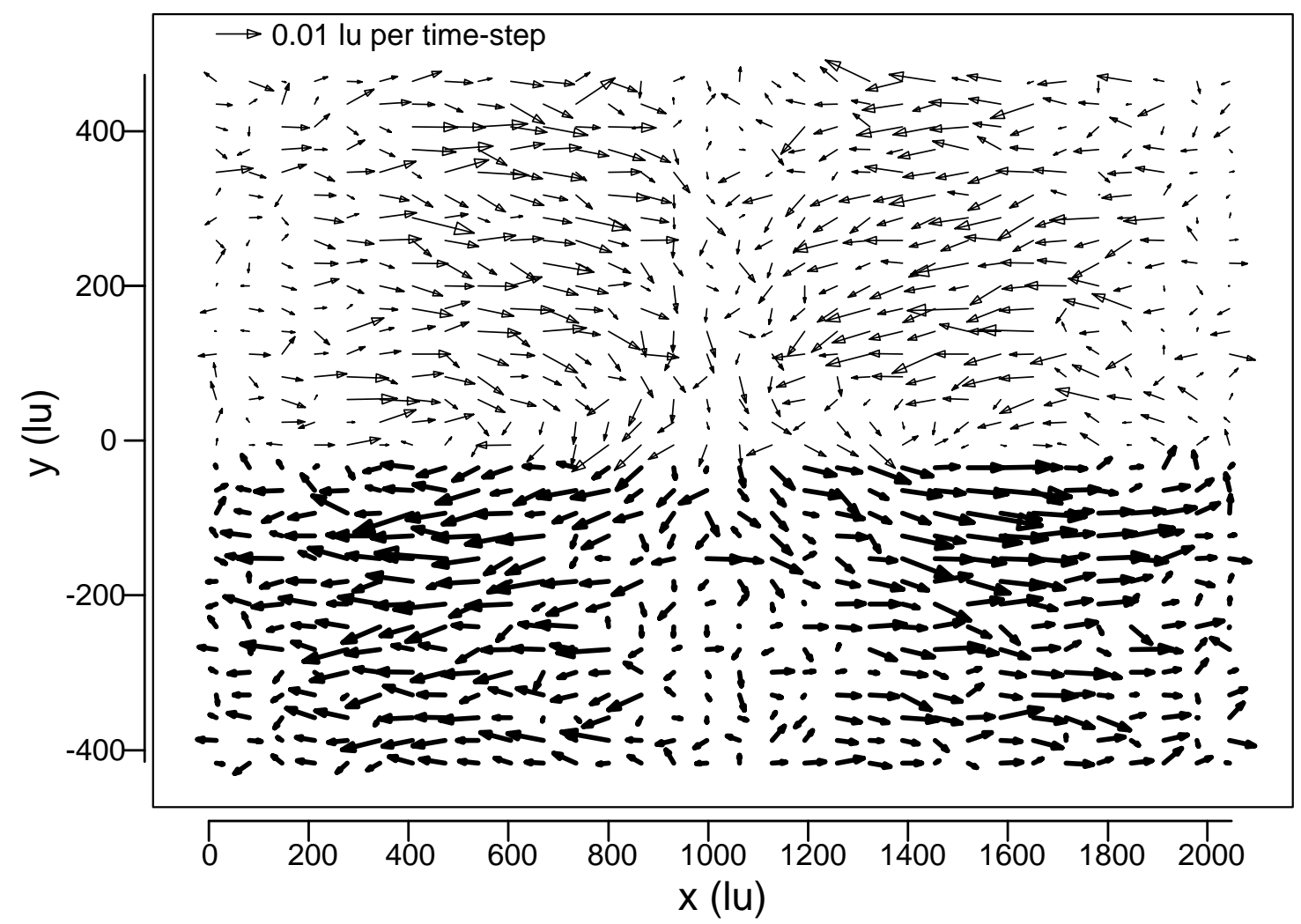

Figure 12: Velocity vector plot for the interfacial internal wave after 4,000 timesteps. Averaging cells containing red particles are represented by the thick arrows. The cells containing only or predominantly blue particles and cells at the boundary containing significant numbers of each particle are represented by the thin arrows. 


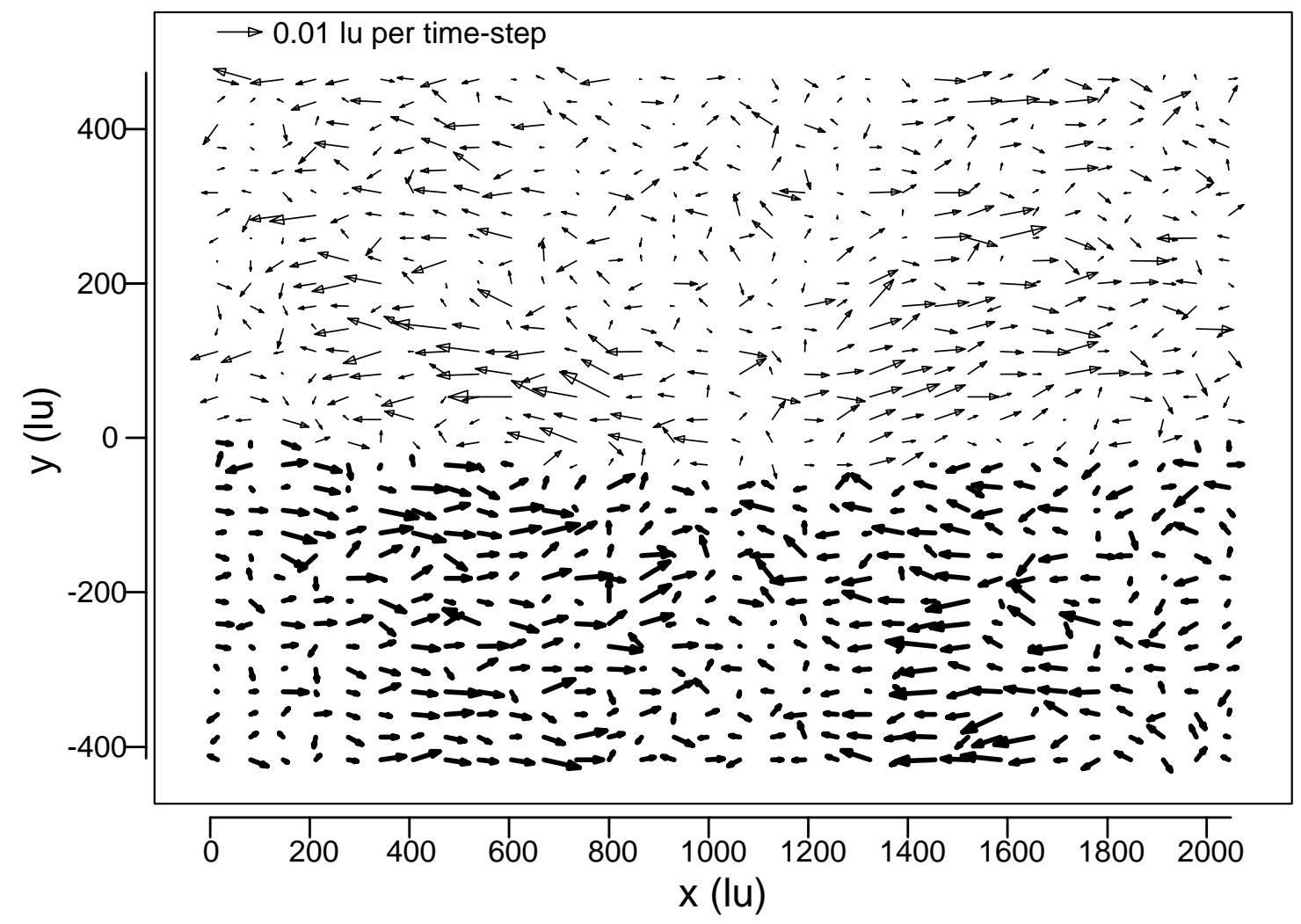

Figure 13: Velocity vector plot for the interfacial internal wave after 10,000 timesteps. Averaging cells containing only or predominantly red particles are represented by the thick arrows. The cells containing only or predominantly blue particles and cells at the boundary containing significant numbers of each particle are represented by the thin arrows. 